Provided for non-commercial research and education use. Not for reproduction, distribution or commercial use.

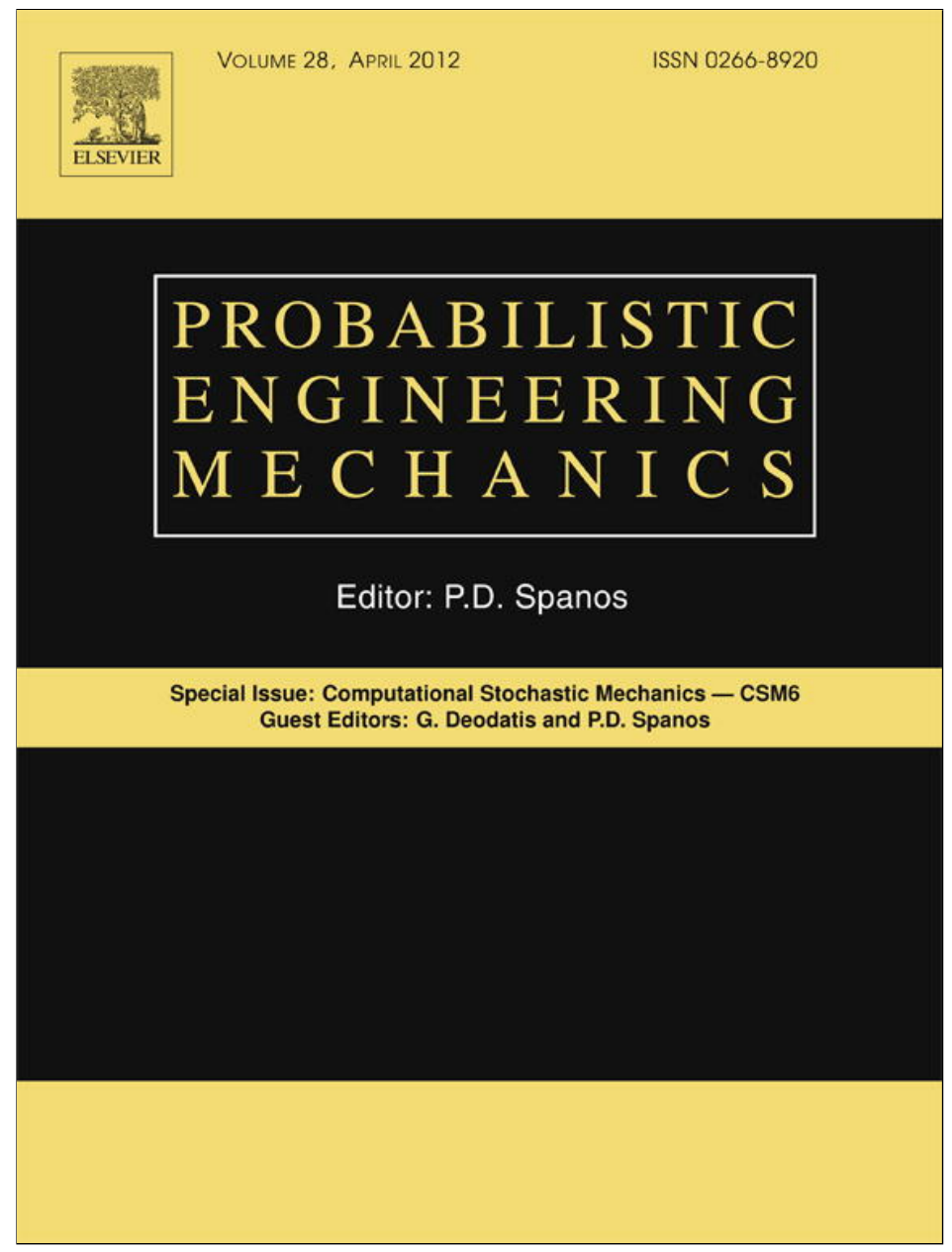

This article appeared in a journal published by Elsevier. The attached copy is furnished to the author for internal non-commercial research and education use, including for instruction at the authors institution and sharing with colleagues.

Other uses, including reproduction and distribution, or selling or licensing copies, or posting to personal, institutional or third party websites are prohibited.

In most cases authors are permitted to post their version of the article (e.g. in Word or Tex form) to their personal website or institutional repository. Authors requiring further information regarding Elsevier's archiving and manuscript policies are encouraged to visit:

http://www.elsevier.com/copyright 


\title{
Stationary and non-stationary stochastic response of linear fractional viscoelastic systems
}

\author{
Mario Di Paola ${ }^{\mathrm{a}}$, Giuseppe Failla ${ }^{\mathrm{b}}$, Antonina Pirrotta ${ }^{\mathrm{a}, *}$ \\ a Dipartimento di Ingegneria Strutturale, Aerospaziale e Geotecnica (DISAG), Università di Palermo, Viale delle Scienze, 90128 Palermo, Italy \\ ${ }^{\mathrm{b}}$ Dipartimento di Meccanica e Materiali (MECMAT), Università di Reggio Calabria, Via Graziella, Località Feo di Vito, 89122 Reggio Calabria, Italy
}

\section{A R T I C L E I N F O}

\section{Article history:}

Received 18 May 2011

Received in revised form

24 June 2011

Accepted 18 August 2011

Available online 7 September 2011

\section{Keywords:}

Viscoelasticity

Fractional calculus

Stochastic input

Non-stationary response

\begin{abstract}
A B S T R A C T
A method is presented to compute the stochastic response of single-degree-of-freedom (SDOF) structural systems with fractional derivative damping, subjected to stationary and non-stationary inputs. Based on a few manipulations involving an appropriate change of variable and a discretization of the fractional derivative operator, the equation of motion is reverted to a set of coupled linear equations involving additional degrees of freedom, the number of which depends on the discretization of the fractional derivative operator. As a result of the proposed variable transformation and discretization, the stochastic analysis becomes very straightforward and simple since, based on standard rules of stochastic calculus, it is possible to handle a system featuring Markov response processes of first order and not of infinite order like the original one. Specifically, for inputs of most relevant engineering interest, it is seen that the response second-order statistics can be readily obtained in a closed form, to be implemented in any symbolic package. The method applies for fractional damping of arbitrary order $\alpha(0 \leq \alpha \leq 1)$. The results are compared to Monte Carlo simulation data.
\end{abstract}

(c) 2011 Elsevier Ltd. All rights reserved.

\section{Introduction}

Fractional calculus [1-3] lends itself to a wide range of applications in science and engineering. Applications in mechanics mainly involve fractional derivatives, now generally recognized as wellestablished tools to model the constitutive behavior of viscoelastic materials. Preliminary investigations in this field trace back to the work by Gemant [4], the first to propose a fractional derivative model for viscoelasticity, and by Scott-Blair and Gaffyn [5]. Later, at the beginning of the 1980s, Bagley and Torvik [6-8] showed that, in order to capture the frequency-dependence of damping properties in viscoelastic materials, fractional derivatives are more appropriate than classical linear models such as the Kelvin-Voigt model, based on which the frequency-dependence is generally overestimated. Further insight into the potential of fractional derivatives as applied to viscoelasticity modeling has been then given by a number of studies in the last three decades [9-14]. In this context, structural engineering applications have been also investigated. Specifically, fractional derivatives have been applied to model viscoelastic dampers [15-18] for vibration and seismic isolation; analytical results have been found in a good agreement with the experimental ones by Makris and Constantinou [19,20].

\footnotetext{
* Corresponding author. Tel.: +39 09123896724 ; fax: +39 0916568407.

E-mail addresses: mario.dipaola@unipa.it (M. Di Paola), giuseppe.failla@unirc.it (G. Failla), antonina.pirrotta@unipa.it (A. Pirrotta).
}

Various strategies have been pursued to compute the deterministic response of single- or multi-degree of freedom systems with a fractional derivative damping. They involve the Laplace transform [7,8], the Fourier transform [21], numerical methods $[15,22,23]$ or an eigenvector expansion $[24,25]$.

For analysis purposes of systems with a fractional derivative damping, in recent years significant research effort has been devoted also to computing the response to stochastic excitations. In this context, a frequency domain approach has been pursued by Spanos and Zeldin [26] and by Rudinger [27]. Alternatively, based on the Laplace transform the system response has been given a time-domain Duhamel integral expression [28] that involves pertinent Green's functions; the latter are available in a closed form for certain values of the fractional derivative order $\alpha$ [2]. A similar approach, where the Duhamel integral is derived based on the Fourier transform of the equation of motion, has been later developed by Kun et al. [29]. Further, the response of a system involving two fractional derivatives has been recently addressed by Huang et al. [30], who derived a Duhamel integral expression by using the Laplace transform in conjunction with the weighted generalized Mittag-Leffler function.

It is now worth remarking that the time-domain methods in Refs. [28-30] provide both stationary and non-stationary response statistics. However, the related analytical expressions do become relatively simple for a Gaussian white noise input only. In the most general case, instead, they involve two-fold integrals in the input 


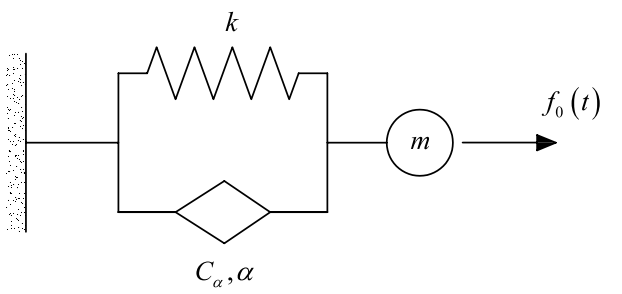

Fig. 1. Linear SDOF system with a fractional derivative damping.

correlation functions, for which only a numerical solution can be sought.

As further, relevant developments in the stochastic analysis of systems with a fractional derivative damping, here also a few recent studies concerning nonlinear systems have to be mentioned. In this regard contributions are due to Huang and Jin [31] and to Spanos and Evangelatos [32]. The first used a classical stochastic averaging procedure for SDOF systems with strongly nonlinear restoring forces and a fractional derivative light damping, subjected to a Gaussian white noise [31]. The second proposed a general frequency domain solution based on statistical linearization; results have been presented for a Duffing oscillator with a fractional derivative damping, subjected to a Gaussian white noise [32].

This paper will focus on linear systems with a fractional derivative damping, subjected to stationary and non-stationary inputs. It will be shown that, for fractional damping of arbitrary order $\alpha(0 \leq \alpha \leq 1)$, the equation of motion can be reverted to a set of coupled linear equations. The latter is built by discretizing the fractional derivative operator, where an appropriate change of variable has been previously introduced. For inputs of most relevant engineering interest, the derived set of linear equations leads to closed-form expressions for the response second-order statistics. It is worth pointing out that, using the proposed variable transformation and discretization, the stochastic analysis of the fractional viscoelastic system becomes straightforward and simple since, relying on standard rules of stochastic calculus, it is possible to handle a system whose responses are Markov processes of first order and not of infinite order like the original one. The advantages of the proposed method with respect to alternative frequency domain $[26,27]$ and time-domain methods [28-30] will be then presented throughout the paper.

\section{Response of fractionally-damped systems}

Let the governing equation of motion of a linear SDOF system equipped with a viscoelastic device be given in the form

$m \ddot{x}(t)+C_{\alpha}\left(D_{0^{+}}^{\alpha} x\right)(t)+k x(t)=f_{0}(t)$,

where $m$ is the mass, $k$ is the stiffness, $C_{\alpha}$ is the damping coefficient, $f_{0}(t)$ is the forcing function and $\left(D_{0^{+}}^{\alpha} x\right)(t)$ is the Riemann-Liouville (RL) fractional derivative governing the damping forces. The system is depicted in Fig. 1: that is, a mass grounded by a linear elastic spring and a fractional derivative damping element.

For $0 \leq \alpha \leq 1$ and for $x(0)=0$ (quiescent system at $t=0$ ), the $\mathrm{RL}$ fractional derivative reads [33]

$$
\left(\mathscr{D}_{0^{+}}^{\alpha} x\right)(t)=\frac{1}{\Gamma(1-\alpha)} \int_{0}^{t} \frac{\dot{x}(\tau)}{(t-\tau)^{\alpha}} \mathrm{d} \tau ; \quad 0 \leq \alpha \leq 1 .
$$

Recognize that, for a quiescent system at $t=0$, the fractional derivative in Eq. (2) coincides with Caputo's fractional derivative. Also, it is known that the values $\alpha=0$ and 1 model the pure solid and the pure fluid state, respectively.
Based on the following relations:

$\frac{1}{\Gamma(1-\alpha)}=\Gamma(\alpha) \frac{\sin (\alpha \pi)}{\pi} ; \quad \Gamma(\alpha)=\int_{0}^{\infty} e^{-z} z^{\alpha-1} \mathrm{~d} z$,

first it is noted that the fractional derivative (2) can be written as

$\left(D_{0^{+}}^{\alpha} x\right)(t)=\frac{\sin (\alpha \pi)}{\pi} \int_{0}^{t} \int_{0}^{\infty} e^{-z} z^{\alpha-1} \frac{\dot{x}(\tau)}{(t-\tau)^{\alpha}} \mathrm{d} z \mathrm{~d} \tau$.

By setting [34]

$z=(t-\tau) y^{2} ; \quad \mathrm{d} z=2(t-\tau) y \mathrm{~d} y$,

Eq. (4) can be then rewritten as

$\left(D_{0^{+}}^{\alpha} x\right)(t)=\mu_{\alpha} \int_{0}^{\infty} \int_{0}^{t} e^{-y^{2}(t-\tau)} \dot{x}(\tau) \mathrm{d} \tau y^{2 \alpha-1} \mathrm{~d} y$,

where $\mu_{\alpha}=2 \sin (\alpha \pi) / \pi$.

Next, denote by $u_{y}(t)$ the Duhamel integral in Eq. (6)

$u_{y}(t)=\int_{0}^{t} e^{-y^{2}(t-\tau)} \dot{x}(\tau) \mathrm{d} \tau$.

For $u_{y}(0)=0, u_{y}(t)$ can be thought of as the response of the following differential equation:

$\dot{u}_{y}(t)+y^{2} u_{y}(t)=\dot{x}(t) ; \quad u_{y}(0)=0$.

The latter governs the response of the Maxwell half oscillator in Fig. 2, having stiffness coefficient equal to $y^{2}$ and a unit damping coefficient, forced by the velocity response of the system in Fig. 1. From Eq. (8) it follows that the RL fractional derivative of $x(t)$, given in Eq. (6), can be rewritten as

$\left(D_{0^{+}}^{\alpha} x\right)(t)=\mu_{\alpha} \int_{0}^{\infty} u_{y}(t) y^{2 \alpha-1} \mathrm{~d} y$.

On the other hand, recognize that Eq. (9) can be reverted to the discrete form

$\left(\mathscr{D}_{0^{+}}^{\alpha} x\right)(t) \approx \mu_{\alpha} \sum_{j=1}^{\infty} u_{y_{j}}(t) y_{j}^{2 \alpha-1} \Delta y$,

where $y_{j}=j \Delta y$ and, due to Eq. (8), $u_{y_{j}}(t)$ is the response of the first order differential equation

$\dot{u}_{y_{j}}(t)+y_{j}^{2} u_{y_{j}}(t)=\dot{x}(t) ; \quad j=1,2, \ldots, \infty$.

At this stage, Eq. (1) can be reverted to the following set of linear ordinary differential equations:

$\ddot{x}(t)+c_{\alpha} \mu_{\alpha} \sum_{j=1}^{\infty} u_{y_{j}}(t) y_{j}^{2 \alpha-1} \Delta y+\omega_{0}^{2} x(t)=f(t) ;$

$\dot{u}_{y_{j}}(t)+y_{j}^{2} u_{y_{j}}(t)=\dot{x}(t), \quad j=1,2, \ldots, \infty$,

where $c_{\alpha}=c_{\alpha} / m, \omega_{0}=\sqrt{k / m}$ and $f(t)=f_{0}(t) / m$. For numerical purposes, obviously a finite number $n$ of terms shall be retained in the first equation of (12). This leads to the linear system

$\dot{\mathbf{z}}=\mathbf{D z}+\mathbf{v} f(t)$,

where

$\mathbf{z}^{T}=\left[\begin{array}{llllll}x & \dot{x} & u_{y_{1}} & u_{y_{2}} & \cdots & u_{y_{n}}\end{array}\right]$

is the vector of state variables, Eq. (15) given in Box I is the coefficient matrix and

$\mathbf{v}^{T}=\left[\begin{array}{llllll}0 & 1 & 0 & 0 & \cdots & 0\end{array}\right]$

is the forcing column vector. Obviously, any time-domain numerical integration scheme can be applied to compute the response of system (13). 


$\mathbf{D}=\left[\begin{array}{llllll}0 & 1 & 0 & 0 & \cdots & 0 \\ -\omega_{0}^{2} & 0 & -c_{\alpha} \mu_{\alpha} y_{1}^{2 \alpha-1} \Delta y & -c_{\alpha} \mu_{\alpha} y_{2}^{2 \alpha-1} \Delta y & \cdots & -c_{\alpha} \mu_{\alpha} y_{n}^{2 \alpha-1} \Delta y \\ 0 & 1 & -y_{1}^{2} & 0 & \cdots & 0 \\ 0 & 1 & 0 & -y_{2}^{2} & \cdots & 0 \\ \cdots & \cdots & \cdots & \cdots & \cdots & \cdots \\ 0 & 1 & 0 & 0 & 0 & -y_{n}^{2}\end{array}\right]$

Box I.

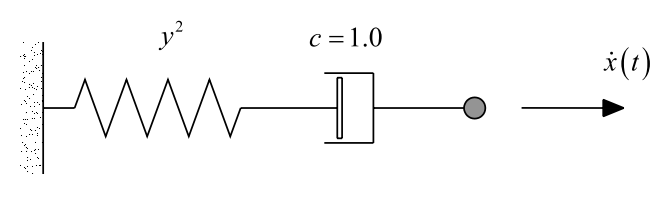

Fig. 2. Maxwell half oscillator forced by the velocity response of the system in Fig. 1.

\section{Stochastic response of fractionally-damped systems}

If the excitation is a stochastic process, the response statistics of system (1) can be computed by applying standard tools of stochastic calculus to system (13). This will be pursued in the following where, as customary, symbols denoting stochastic processes will be labeled with capital letters; that is, $f(t)$ will be replaced by $F(t), \mathbf{z}(t)$ by $\mathbf{Z}(t)$.

Then, let $\mathbf{Z}(t)$ be the response vector of system (13) to a stochastic input $F(t)$. The latter is assumed to have, for simplicity, zero mean. Based on the coordinate transformation

$\mathbf{Z}(t)=\boldsymbol{\Phi} \mathbf{Q}(t)$,

where $\boldsymbol{\Phi}$ is the matrix of the eigenvectors of matrix $\mathbf{D}$ in Eq. (15), Eq. (13) may be rewritten in the form

$\dot{\mathbf{Q}}=\mathbf{\Lambda} \mathbf{Q}+\boldsymbol{\Phi}^{-1} \mathbf{v} F(t)$,

where

$\Lambda=\boldsymbol{\Phi}^{-1} \mathbf{D} \boldsymbol{\Phi}$

is the diagonal matrix whose non-zero elements $\Lambda_{j j}$ are the eigenvalues of system (13). It may be easily verified that, for $c_{\alpha}>0$, only two eigenvalues are complex (and conjugate) while the remaining are real and approximately equal to the diagonal elements $y_{j}^{2}$ in matrix $\mathbf{D}$, that is $\Lambda_{j j} \approx y_{j}^{2}$.

For system (18) the time-dependent matrix of the secondorder response statistics, $\boldsymbol{\Xi}(t)=E\left[\mathbf{Q Q}^{T}\right]$, can be computed by integrating the uncoupled Lyapunov moment equations

$\dot{\Xi}(t)=\Lambda \Xi(t)+\boldsymbol{\Xi}(t) \boldsymbol{\Lambda}+\mathbf{G}(t)$,

with $\mathbf{G}(t)$ being

$\mathbf{G}(t)=\boldsymbol{\Phi}^{-1} \mathbf{v} E\left[F(t) \mathbf{Q}^{T}(t)\right]+E[\mathbf{Q}(t) F(t)]\left(\boldsymbol{\Phi}^{-1} \mathbf{v}\right)^{T}$.

Upon solving Eq. (20) for $\boldsymbol{\Xi}(t)$, the variances of the displacement and the velocity response of the original system (1) can be finally written, respectively, as

$\sigma_{X X}^{2}(t)=\sum_{j, k}^{n} \Phi_{1 j} \Phi_{1 k} \Xi_{j k}(t) ;$

$\sigma_{\dot{X}}^{2}(t)=\sum_{j, k}^{n} \Phi_{2 j} \Phi_{2 k} \Xi_{j k}(t)$,

while the cross-covariance is given as

$\sigma_{X \dot{X}}^{2}(t)=\sum_{j, k}^{n} \Phi_{1 j} \Phi_{2 k} \Xi_{j k}(t)$.
In Eqs. (22) $\Phi_{1 j}$ and $\Phi_{2 j}$ denote the $j$-th element of the first and second row of matrix $\boldsymbol{\Phi}$, while $\Xi_{j k}$ denotes the element at row $j$ and column $k$ of matrix $\boldsymbol{\Xi}(t)$.

Closed-form solutions to the Lyapunov moment equations (20) of the uncoupled system (18) do exist in many cases of engineering interest. For instance if $F(t)=W(t)$, where $W(t)$ is a Gaussian white noise with two-sided power spectral density $S_{0}$, that is $E[W(t) W(t+\tau)]=2 \pi S_{0} \delta(\tau)$, for zero initial conditions it readily follows that

$\Xi_{j k}(t)=\frac{G_{j k}}{\Lambda_{j j}+\Lambda_{k k}}\left[e^{\left(\Lambda_{j j}+\Lambda_{k k}\right) t}-1\right] ; j, k=1, \ldots, n$,

where $G_{j k}$ is the element at row $j$ and column $k$ of the $n \times n$ matrix

$\mathbf{G}=2 \pi S_{0} \boldsymbol{\Phi}^{-1} \mathbf{v}\left(\boldsymbol{\Phi}^{-1} \mathbf{v}\right)^{T}$.

Similarly, if $F(t)$ is a uniformly-modulated white noise $F(t)=$ $A(t) W(t)$, where $A(t)$ is a time-dependent modulating function, for which $E[F(t) F(t+\tau)]=2 \pi S_{0} A^{2}(t) \delta(\tau)$ holds, $\Xi_{j k}(t)$ is given by

$$
\begin{aligned}
\Xi_{j k}(t)= & e^{\left(\Lambda_{j j}+\Lambda_{k k}\right) t}\left(\int_{1}^{t} e^{-\left(\Lambda_{j j}+\Lambda_{k k}\right) \tau} G_{j k}(\tau) \mathrm{d} \tau\right. \\
& \left.-\int_{1}^{0} e^{-\left(\Lambda_{j j}+\Lambda_{k k}\right) \tau} G_{j k}(\tau) \mathrm{d} \tau\right) ; \quad j, k=1, \ldots, n,
\end{aligned}
$$

where $G_{j k}(t)$ are time-dependent elements of the matrix

$\mathbf{G}(t)=2 \pi S_{0} A^{2}(t) \boldsymbol{\Phi}^{-1} \mathbf{v}\left(\boldsymbol{\Phi}^{-1} \mathbf{v}\right)^{T}$.

It is recognized that closed-form solutions to Eq. (25) can be readily found, for instance, for any amplitude modulating function $A(t)$ of exponential form, as generally encountered in seismic engineering applications. Note also that, still for non-stationary inputs, further closed-form solutions to the Lyapunov moment equations (20) can be derived as shown in the following Section 4.2.

It is now worth remarking that, based on alternative timedomain methods existing in the literature, the response statistics are generally obtained, at each time instant, upon computing a two-fold integral involving the input correlation function and appropriate kernel functions depending on the system parameters [28-30]. In general, such two-fold integrals have to be evaluated numerically. The proposed method, instead, upon performing a preliminary modal analysis to set the uncoupled Eqs. (18), provides the response statistics (22) in an analytical form, based on the closed-form solutions to the Lyapunov moment equations (20). Note that the analytical form may allow one to carry out, in a very efficient manner, a sensitivity analysis on input parameters such as, for instance, the parameters of a modulating function of the input (see Eq. (25) and Eq. (29) through Eq. (32) in Section 4.2). On the other hand, as shown by the numerical applications in Section 4, a limited number of additional half-oscillators is generally required in system (13) to achieve a reasonable accuracy. This may allow, on average, computational savings up to $30 \%$ with respect to the time-domain methods in Refs. [28-30]. 
Finally, it has to be noted that the proposed method allows one to describe both the stationary and non-stationary response; obviously, if the stationary response is of interest, $\dot{\Xi}(t)=0 \forall t$ shall be set in Eq. (20). It is recognized that frequency domain methods, although very efficient from a computational point of view, can capture the stationary response only $[26,27]$.

\section{Numerical applications}

Two examples are now considered to assess the accuracy of the proposed method. First it will be applied to build the statistics of the transient and stationary response to a Gaussian white noise, then to build the statistics of the non-stationary response to a uniformly-modulated non-stationary seismic input.

\subsection{Response to white noise}

Let $F(t)=W(t)$ be a zero-mean Gaussian white noise of twosided power spectral density $S_{0}=(2 \pi)^{-1}$. As shown in Section 3 , in this case, the second-order response statistics can be computed based on Eq. (22), where $\Xi_{j k}(t)$ is given by Eq. (23).

To implement the proposed method, the $y$-axis (see Eq. (10)) is discretized in two subintervals: the first is [0,0.05] and is divided into 200 equal steps; the second is $[0.05,20.0]$ and is divided into 100 steps. Therefore, a total number $n=300$ of additional oscillators is considered to build system (13). The above discretization parameters are selected to provide an accurate description of the integrand function $e^{-z} z^{\alpha-1}$ in Eq. (3), taking into account that $z=(t-\tau) y^{2}$.

The following parameters are considered in Eq. (1): $\omega_{0}=$ $1.0 ; c_{\alpha}=1.0 ; \alpha=0.3$ and $\alpha=0.5$. For an individual sample of the white noise input, Figs. 3 and 4 show the displacement response as obtained by the proposed method (PM) and by integrating Eq. (1) via the central difference method. Specifically, to build the response by the PM Eqs. (13) are integrated based on a fourth-order Runge-Kutta algorithm; to build the response by the central difference method the fractional derivative is reverted to a finite sum by using the Riemann-Liouville (RL) approximation [35]. A very satisfactory agreement is found between the two solutions, for both $\alpha=0.3$ and 0.5 . Next, Figs. 5 through 8 show the variances of the displacement and velocity responses on the time interval $[0,20]$, as obtained by the closed-form solutions (22) and by a digital simulation with 5000 samples, the latter being built by integrating Eq. (1) via the central difference method in conjunction with an RL approximation of the fractional derivative. Again, a very good agreement is found across the whole time range of interest, for both $\alpha=0.3$ and 0.5 .

\subsection{Response to a non-stationary input}

Next, let $F(t)$ be a zero-mean non-stationary process belonging to the class of Priestley's oscillatory processes [36], that is

$F(t)=A(t) \bar{F}(t)$,

where $A(t)$ is a time-dependent amplitude modulating function and $\bar{F}(t)$ is a stationary process of two-sided power spectral density $S(\omega)$.

In general, for the process $F(t)$ the spectral representation [37]

$F(t) \approx A(t) \sum_{l=1}^{M} \sqrt{4 S\left(\omega_{l}\right) \Delta \omega} \cos \left(\omega_{l} t+\varphi_{l}\right)$

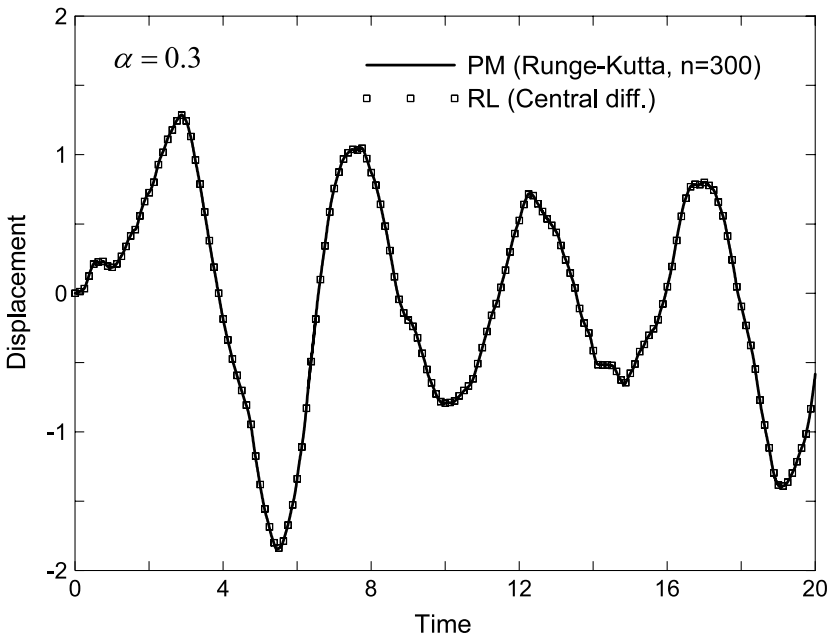

Fig. 3. Displacement response to a Gaussian white noise sample, for $\alpha=0.3$.

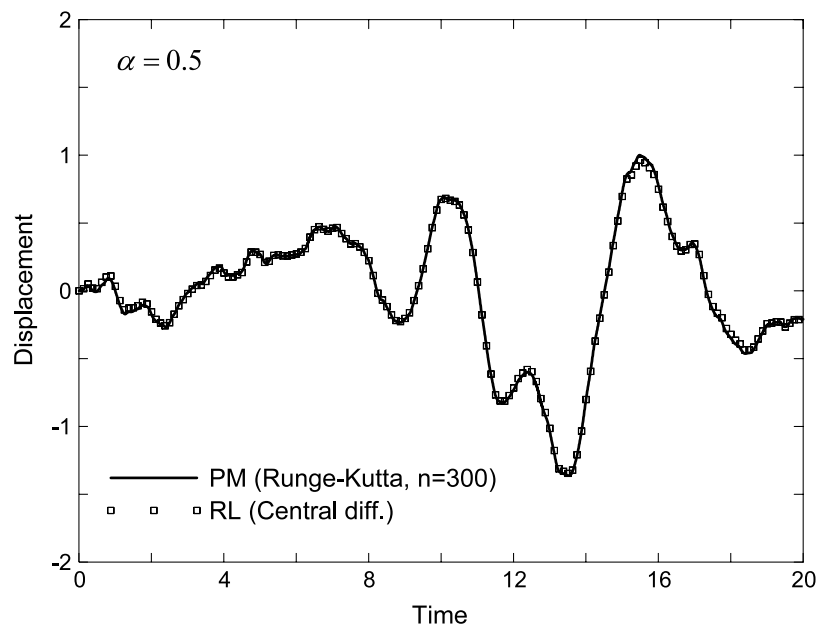

Fig. 4. Displacement response to a Gaussian white noise sample, for $\alpha=0.5$.

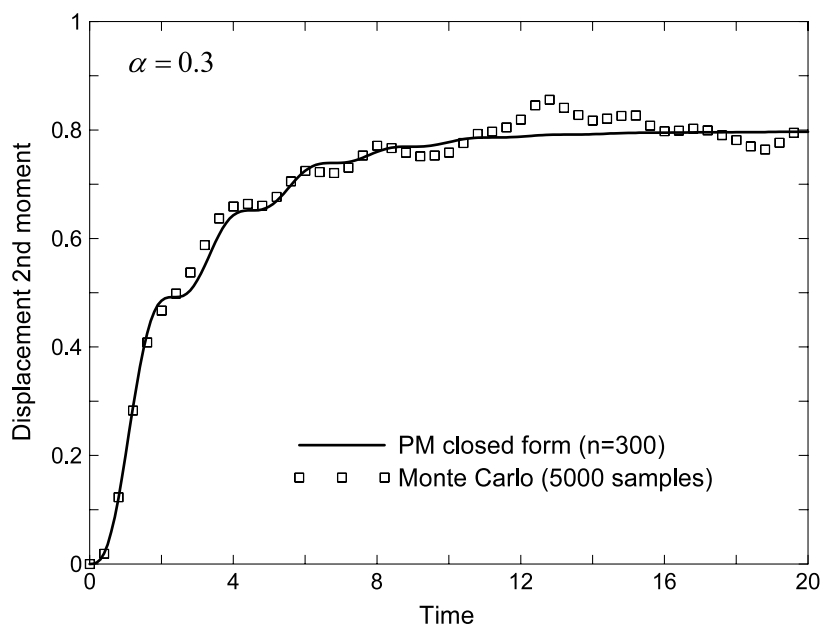

Fig. 5. Displacement variance under a Gaussian white noise, for $\alpha=0.3$.

can be adopted, where $\varphi_{l}$ are $M$ realizations of a random variable uniformly distributed in $[0,2 \pi]$. It can be seen that, if $A(t)$ in Eq. (27) is given the exponential form

$A(t)=e^{-a_{1} t}-e^{-a_{2} t}$,

upon replacing Eq. (28) for $F(t)$ in Eq. (18), closed-form solutions can be found to the Lyapunov moment equations (20). They are 


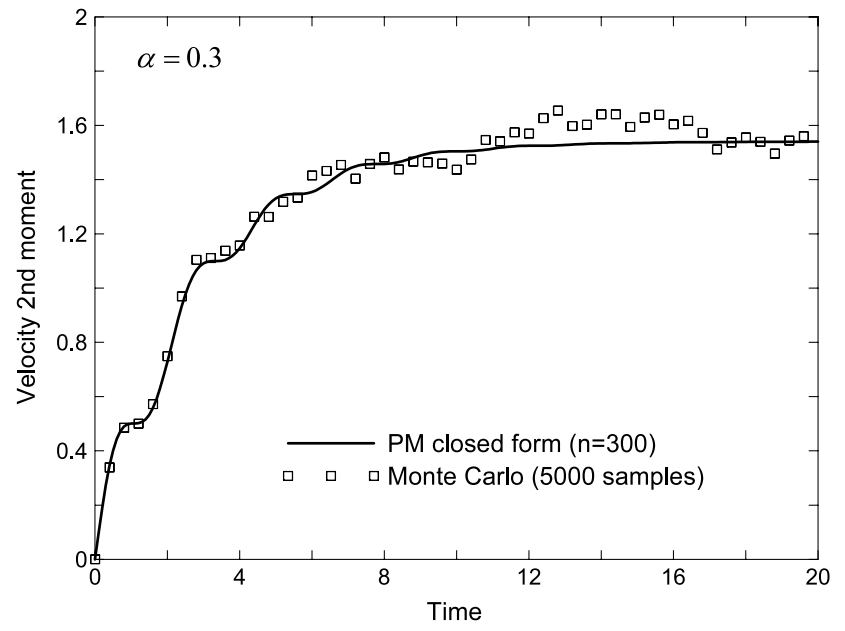

Fig. 6. Velocity variance under a Gaussian white noise, for $\alpha=0.3$.

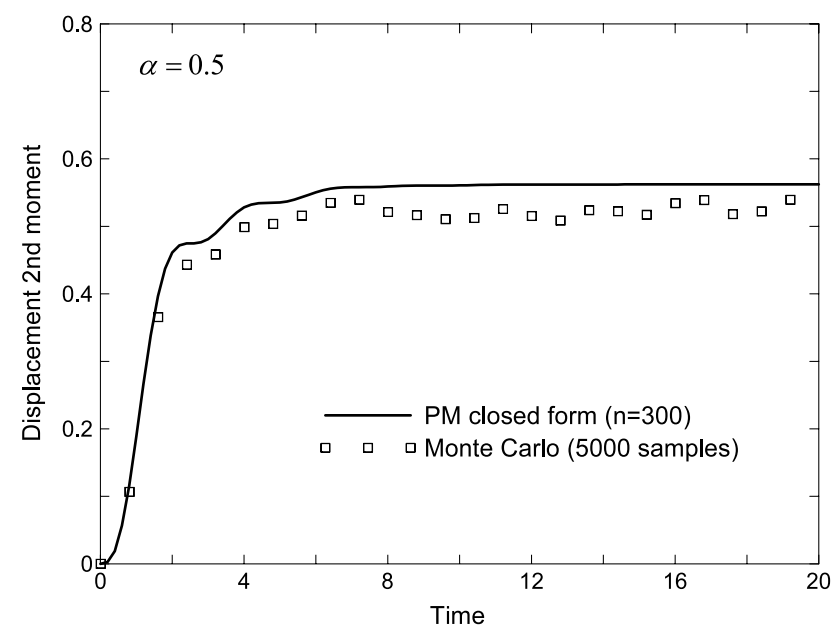

Fig. 7. Displacement variance under a Gaussian white noise, for $\alpha=0.5$.

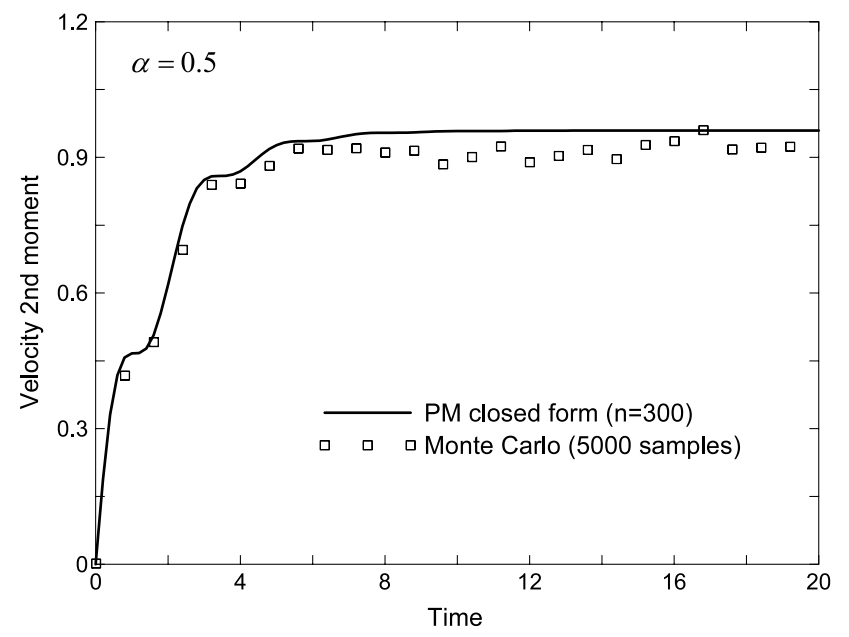

Fig. 8. Velocity variance under a Gaussian white noise, for $\alpha=0.5$.

given as

$$
\begin{aligned}
& E\left[Q_{i}^{2}\right]=r_{i}^{2} \sum_{m, q=1}^{2}(-1)^{m+q+1} \sum_{l=1}^{M} 4 S\left(\omega_{l}\right) \Delta \omega\left[h_{1}-h_{2}-h_{3}\right] \cdot h_{4} ; \\
& \quad i=1,2, \ldots, n,
\end{aligned}
$$

and

$$
\begin{aligned}
E\left[Q_{i} Q_{k}\right]= & \sum_{\substack{i, k=1 \\
i \neq k}}^{2} r_{i} r_{k} \sum_{m, q=1}^{2}(-1)^{m+q+1} \sum_{l=1}^{M} 4 S\left(\omega_{l}\right) \Delta \omega \\
& \times\left[h_{1}-h_{2}-h_{3}\right] \cdot h_{4} ; \quad i \neq k ; i, k=1,2, \ldots, n,
\end{aligned}
$$

where $r_{i}$ is the $i$ th component of vector $\boldsymbol{\Phi}^{-1} \mathbf{v}$; also, $h_{1}, h_{2}, h_{3}$ and $h_{4}$ are the functions

$$
\begin{aligned}
& h_{1}\left(a_{m}, a_{q}, \lambda_{i k}, \Lambda_{k k}, \omega_{l}, t\right) \\
& =e^{-\left(a_{m}+a_{q}\right) t}\left(a_{m}+\lambda_{i k}-\Lambda_{k k}\right)\left(a_{q}+\Lambda_{k k}\right) \\
& \quad \times\left[-a_{m}-\lambda_{i k}+\Lambda_{k k}-e^{\left(a_{m}+a_{q}+\lambda_{i k}\right) t}\left(a_{q}+\Lambda_{k k}\right)\right. \\
& \left.\quad+e^{\left(a_{q}+\lambda_{i k}\right) t}\left(a_{m}+a_{q}+\lambda_{i k}\right) \cos \left(\omega_{l} t\right)\right] ; \\
& h_{2}\left(a_{m}, a_{q}, \lambda_{i k}, \Lambda_{k k}, \omega_{l}, t\right) \\
& =e^{\left(a_{q}+\Lambda_{k k}\right) t}\left(a_{m}+a_{q}+\lambda_{i k}\right)\left(a_{q}-a_{m}+2 \Lambda_{k k}-\lambda_{i k}\right) \\
& \quad \times \omega_{l} \sin \left(\omega_{l} t\right) ; \\
& h_{3}\left(a_{m}, a_{q}, \lambda_{i k}, \Lambda_{k k}, \omega_{l}, t\right) \\
& =\omega_{l}^{2}\left[a_{q}+e^{\left(a_{m}+a_{q}+\lambda_{i k}\right) t}\left(a_{m}+\lambda_{i k}-\Lambda_{k k}\right)\right. \\
& \left.\quad+\Lambda_{k k}-e^{\left(a_{q}+\Lambda_{k k}\right) t}\left(a_{m}+a_{q}+\lambda_{i k}\right) \cos \left(\omega_{l} t\right)\right] ; \\
& h_{4}\left(a_{m}, a_{q}, \lambda_{i k}, \Lambda_{k k}, \omega_{l}\right) \\
& =\left\{2\left(a_{m}+a_{q}+\lambda_{i k}\right)\left[\left(a_{m}+\lambda_{i k}-\Lambda_{k k}\right)^{2}+\omega_{l}^{2}\right]\right. \\
& \left.\quad \times\left[\left(a_{q}+\Lambda_{k k}\right)^{2}+\omega_{l}^{2}\right]\right\}^{-1}
\end{aligned}
$$

for $\lambda_{i k}=\Lambda_{i i}+\Lambda_{k k}$. Based on Eqs. (32), the response statistics (22) can then be given a closed-form expression.

As in the previous case, the proposed method is implemented by discretizing the $y$-axis (see Eq. (10)) in two subintervals: the first is $[0,0.05]$ and is divided into 200 equal steps, the second is $[0.05,20.0]$ and is divided into 100 steps. Therefore, again a total number $n=300$ of additional oscillators is considered to build system (13). The following parameters are set in Eq. (1): $\omega_{0}=1.0 ; c_{\alpha}=1.0$ and $\alpha=0.5 ; a_{1}=0.25$ and $a_{2}=0.5$ are selected for the amplitude modulating function (28), while $S(\omega)$ is given the form

$S(\omega)=\frac{\left(\omega / \omega_{s}\right)^{2}}{\left\{\left[1-\left(\omega / \omega_{s}\right)^{2}\right]^{2}+\left(2 \zeta \omega / \omega_{s}\right)^{2}\right\}^{2}}$,

where $\omega_{s}=12.0$ and $\zeta=0.4$. In Eq. (28) for $F(t), \Delta \omega=0.05$ and $M=600$ are selected.

Figs. 9 and 10 show the variances of the displacement and velocity response on the time interval $[0,40]$, as obtained by the closed-form solutions (22) and by a Monte Carlo simulation with 5000 samples, the latter being built by integrating Eq. (1) via the central difference scheme with an RL approximation for the fractional derivative [35]. A very satisfactory agreement is encountered between the PM and the Monte Carlo solution, across the whole time range of interest.

\section{Conclusions}

A method to compute the stochastic response of fractionallydamped SDOF systems has been presented. The key idea is to discretize the fractional operator based on an appropriate change of variable. In this manner, the original equation of motion can be reverted to a set of equations involving additional oscillators, the 


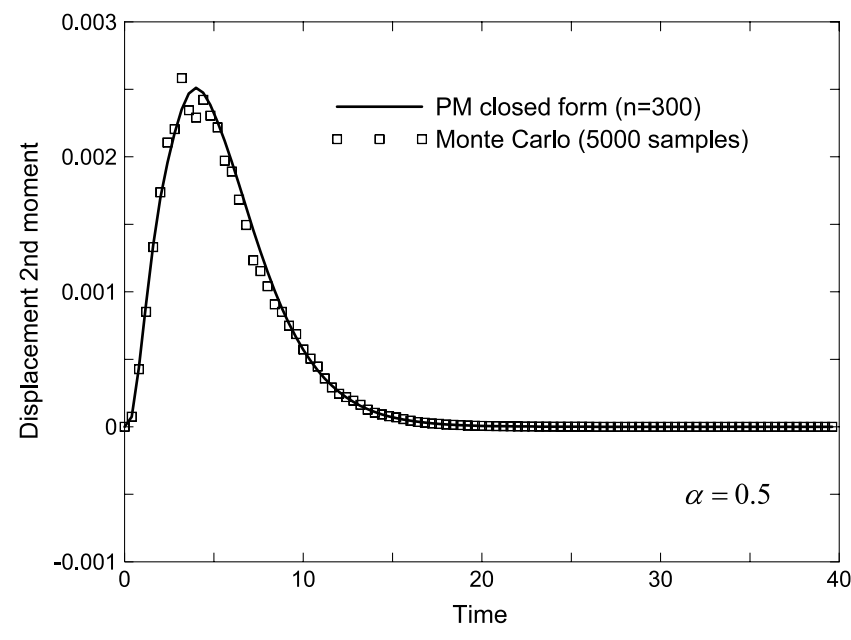

Fig. 9. Displacement variance under a non-stationary input, for $\alpha=0.5$.

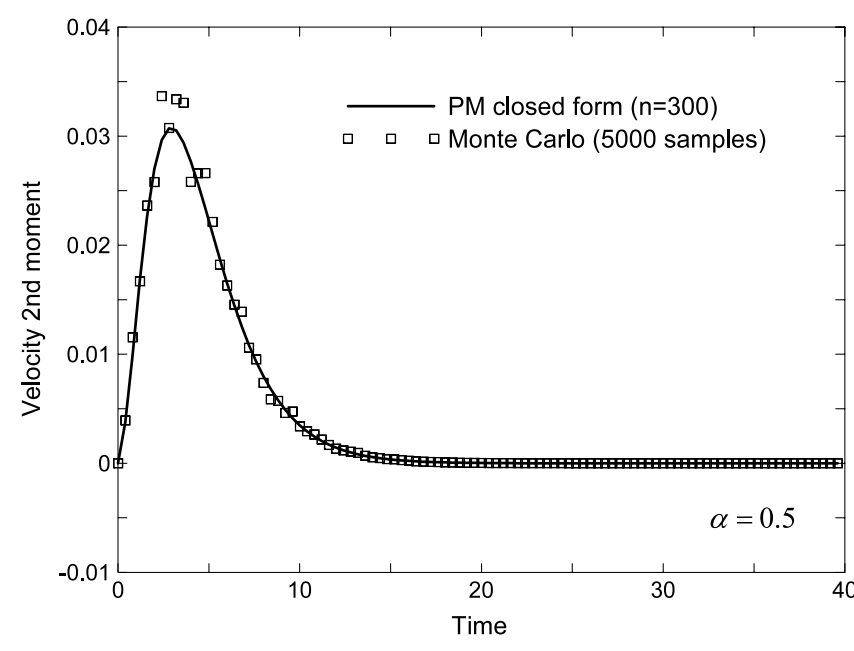

Fig. 10. Velocity variance under a non-stationary input, for $\alpha=0.5$.

number of which depends on the adopted discretization. Examples have been given of stationary and non-stationary stochastic inputs for which the method leads to closed-form expressions of the response second-order statistics, readily implementable in any symbolic package. The numerical applications show that, in general, a limited number of additional oscillators is required to achieve accurate results as compared to Monte Carlo simulation data.

\section{References}

[1] Oldham KB, Spanier J. The fractional calculus. New York: Academic Press; 1974.

[2] Miller KS, Ross B. An introduction to the fractional calculus and fractional differential equations. New York: John Wiley and Sons; 1993.

[3] Gorenflo R, Mainardi F. Fractals and fractional calculus in continuum mechanics. In: Carpinteri A, Mainardi F, editors. Fractional calculus: Integral and differential equations of fractional order. Wien, New York: SpringerVerlag; 1997. p. 223-76.

[4] Gemant A. On Fractional Differentials. Philosophical Magazine Series, vol. 25. 1938. p. $540-9$.

[5] Scott-Blair GW, Gaffyn JE. An application of the theory of quasi-properties to the treatment of anomalous strain-stress relations. The Philosophical Magazine 1949;40:80-94.

[6] Bagley RL, Torvik PJ. A theoretical basis for the application of fractional calculus to viscoelasticity. Journal of Rheology 1983;27:201-10.
[7] Bagley RL, Torvik PJ. Fractional calculus - a different approach to the analysis of viscoelastically damped structures. American Institute of Aeronautics and Astronautics Journal 1983;21:741-8.

[8] Bagley RL, Torvik PJ. Fractional calculus in the transient analysis of viscoelastically damped structures. American Institute of Aeronautics and Astronautics Journal 1985;23:918-25.

[9] Rogers L. Operators and fractional derivatives for viscoelastic constitutive equations. Journal of Rheology 1983;27(4):351-72.

[10] Koeller RC. Application of fractional calculus to the theory of viscoelasticity. Journal of Applied Mechanics 1984;51:299-307.

[11] Pritz T. Analysis of four-parameter fractional derivative model of real solid materials. Journal of Sound and Vibration 1996;195:103-15.

[12] Bossemayer H. Evaluation technique for dynamic moduli. Mechanics TimeDependent Materials 2001;5:273-91.

[13] Galucio AC, Deu JF, Ohayon R. Finite element formulation of viscoelastic sandwich beams using fractional derivative operators. Computational Mechanics 2004;33:282-91.

[14] Di Paola M, Pirrotta A, Valenza A. Visco-elastic behavior through fractional calculus: an easier method for best fitting experimental results. Mechanics of Materials 2011, in press (doi:10.1016/j.mechmat.2011.08.016).

[15] Koh CG, Kelly LM. Application of fractional derivatives to seismic analysis of base isolated models. Earthquake Engineering and Structural Dynamics 1990; 19(2):229-41.

[16] Lee HH, Tsai CS. Analytical model for viscoelastic dampers for seismic mitigation of structures. Computers and Structures 1994;50(1):111-21.

[17] Shen KL, Soong TT. Modeling of viscoelastic dampers for structural applications. Journal of Engineering Mechanics 1995;121:694-701.

[18] Papoulia KD, Kelly JM. Visco-hyperelastic model for filled rubbers used in vibration isolation. Journal of Engineering Materials and Technology 1997; 119:292-7.

[19] Makris N, Constantinou MC. Fractional-derivative Maxwell model for viscous dampers. Journal of Structural Engineering 1991;117:2708-24.

[20] Makris N, Constantinou MC. Spring-viscous damper systems for combined seismic and vibration isolation. Earthquake Engineering and Structural Dynamics 1992;21(8):649-64.

[21] Gaul L, Klein P, Kemple S. Impulse response function of an oscillator with fractional derivative in damping description. Mechanics Research Communications 1989;16:297-305.

[22] Lixia Y, Agrawal OP. A numerical scheme for dynamic systems containing fractional derivatives, In: Proceedings of 1998 ASME Design Engineering Technical Conferences, September 13-16, Atlanta, Georgia, 1998.

[23] Shokooh A, Suarez L. A comparison of numerical methods applied to a fractional model of damping materials. Journal of Vibration and Control 1999; 5:331-54.

[24] Suarez L, Shokooh A. An eigenvector expansion method for the solution of motion containing fractional derivatives. Journal of Applied Mechanics 1997; 64:629-35.

[25] Chang TS, Singh MP. Seismic analysis of structures with a fractional derivative model of viscoelastic dampers. Earthquake Engineering and Engineering Vibration 2002;1(2):251-60.

[26] Spanos PD, Zeldin BA. Random vibration of systems with frequency-dependent parameters or fractional derivatives. Journal of Engineering Mechanics 1997; 123:290-2.

[27] Rudinger F. Tuned mass damper with fractional derivative damping. Engineering Structures 2006;28:1774-9.

[28] Agrawal OP. Stochastic analysis of dynamic systems containing fractional derivatives. Journal of Sound and Vibration 2001;247(5):927-38.

[29] Kun Y, Li L, Jiaxiang T. Stochastic seismic response of structures with added viscoelastic dampers modeled by fractional derivative. Earthquake Engineering and Engineering Vibration 2003;2(1):133-9.

[30] Huang ZL, Jin XL, Lim CW, Wang Y. Statistical analysis for stochastic systems including fractional derivatives. Nonlinear Dynamics 2010;59: 339-349.

[31] Huang ZL, Jin XL. Response and stability of a SDOF strongly nonlinear stochastic system with light damping modeled by a fractional derivative. Journal of Sound and Vibration 2009;319:1121-35.

[32] Spanos PD, Evangelatos GI. Response of a non-linear system with restoring forces governed by fractional derivatives - Time domain simulation and statistical linearization solution. Soil Dynamics and Earthquake Engineering 2010;30:811-21.

[33] Podlubny I. Fractional differential equations. New York: Academic Press; 1999.

[34] Schmidt A Gaul L On a critique of a numerical scheme for the calculation of fractionally damped dynamical systems. Mechanics Research Communications 2006;33:99-107.

[35] Schmidt A, Gaul L. On the numerical evaluation of fractional derivatives in multi-degree-of-freedom systems. Signal Processing 2006;86:2592-601.

[36] Priestley MB. Spectral analysis and time series. New York: Academic Press: 1981.

[37] Shinozuka M, Deodatis G. Stochastic process models for earthquake ground motion. Probabilistic Engineering Mechanics 1988;3(3):114-23. 